\title{
The Genetic Analysis of Carbohydrate Utilization in Aspergillus nidulans
}

\author{
By C. F. ROBERTS* \\ Department of Genetics, The University, Glasgow
}

(Received 23 July 1962)

\begin{abstract}
SUMMARY
The isolation and genetic analysis of a new class of mutants of the mould Aspergillus nidulans is described. The mutants were detected by their inability to utilize specific carbohydrates as sole carbon source for growth. All of the mutants are recessive and analysis of 27 mutants has resulted in the description of 10 new loci concerned with the utilization of carbohydrates in the organism; the loci have been allocated to linkage groups and 6 were mapped meiotically. Two loci which control lactose utilization are not linked and neither are 5 loci which control the utilization of galactose. A series of sorbitol mutants is unusual in that they are all non-complementary when combined in heterokaryons, although certain pairs of mutants complement in the corresponding heterozygous diploids.
\end{abstract}

\section{INTRODUCTION}

It is a conspicuous omission in fungal genetics that although auxotrophic mutants have been isolated in many filamentous fungi and it is known that in general these fungi metabolize a wide range of carbohydrates, very few attempts to isolate mutants defective in carbohydrate utilization have been reported (Emerson, 1944; Landman, 1950). Mutants defective either in the ability to form specific carbohydrases or carbohydrate permeases (Cohen \& Monod, 1957) may be identified by their failure to grow with particular carbohydrates as sole carbon source. The present paper describes the isolation and genetic analysis of mutants of this type in the homothallic ascomycete Aspergillus nidulans.

\section{METHODS}

Strains and media. The strains of Aspergillus nidulans used were from the stock held in the Department of Genetics of Glasgow University. Media and routine methods for $A$. nidulans described by Pontecorvo et al. (1953) were used. Minimal medium is a solution of inorganic salts, including nitrate and sulphate as nitrogen and sulphur sources, with $1 \%(\mathrm{w} / \mathrm{v})$ glucose as carbon source. Complete medium is a complex medium containing casein hydrolysate, yeast extract, some vitamins, a hydrolysate of yeast nucleic acid, and salts and glucose as in minimal medium. Basal medium, used in the isolation of the sugar mutants and in classifying strains by their ability to utilize different sugars for growth, is minimal medium without a carbon source. The media were solidified by adding $1.5 \%(\mathrm{w} / \mathrm{v})$ unwashed Difco agar. Sugars were sterilized separately by autoclaving $\left(115^{\circ}\right.$ for $10 \mathrm{~min}$.) in

\footnotetext{
* Present address : Microbiology Unit, Department of Biochemistry, University of Oxford.
} 
distilled water and were added to molten basal medium to a final concentration of $1 \%(\mathrm{w} / \mathrm{v})$. The sugars used were $\mathrm{D}$-glucose, lactose and sucrose (all analytical reagents) and $\mathrm{D}$-fructose (biochemical reagent) from British Drug Houses Ltd.; $\mathrm{D}$-galactose, maltose and $\mathrm{D}$-sorbitol (all bacteriological reagents) from T. Kerfoot, Vale of Bardsley, Lancs.

Isolation of mutants. The mutants were isolated by a replica plating technique (Roberts, 1959) following ultraviolet (u.v.)-irradiation. Suspensions of mainly separate conidia of Aspergillus nidulans strains bi1; w3 (biotinless; white conidia) or $y 2 ;$ pyro4 (yellow conidia; pyridoxinless), were exposed to u.v. radiation until the viable count was decreased to about $5 \%$ of the original value; the suspensions were then diluted and plated on complete medium to yield 100 to 150 colonies on each plate. The plates were incubated until good sporulation had occurred, when conidia were replicated on to a series of seven plates. Five were test plates containing basal medium supplemented with either biotin or pyridoxin (required by either parent strain), and to each of which either galactose, lactose, maltose, sorbitol or sucrose had been added as sole source of carbon: the sixth was a control containing minimal medium (glucose) supplemented with either biotin or pyridoxin, and the seventh plate contained complete medium.

Auxotrophic mutants with a nutritional requirement in addition to that of the parent strain were detected by their failure to grow on any of the five test plates or on supplemented minimal medium, though growing on complete medium. Sugar mutants were identified by their failure to grow on one or more of the test plates although growing on supplemented minimal medium and on complete medium. Mutants were isolated from the complete medium plate, purified by single colony isolation and their phenotypes retested. Only strains clearly different from the wild-type in their growth on the relevant sugar (though growing as well as the parent strain on glucose) were retained for analysis. When more than one mutant was isolated from the same set of plates only one was retained unless they were clearly different phenotypically.

Genetic analysis of mutants. Phenotypically similar mutants were separated into functional groups by complementation tests (Pontecorvo, 1952 $a$; 1958) made with the appropriate heterozygous diploids (Roper, 1952). Location of the mutants in the linkage maps of Aspergillus nidulans was achieved in two stages; first, identification of the linkage group into which a mutant fell and, secondly, location of the mutant in relation to other markers in that group. The first stage of location used the parasexual cycle (Pontecorvo, 1954) and the technique of mitotic haploidization (Pontecorvo, Tarr Gloor \& Forbes, 1954; Pontecorvo \& Käfer, 1958) combined with the use of 'tester strains' (Forbes, 1959) marked in each of the eight linkage groups of $A$. nidulans (Käfer, 1958; Elliott, 1960). The second stage of location used the sexual cycle and the technique of perithecium analysis (Hemmons, Pontecorvo \& Bufton, 1953). Methods given by Mather (1951) were followed in the detection and estimation of linkage.

Markers used in the present work. The markers used in the analysis are listed at the bottom of Figs. 1 and 2; for further details see Pontecorvo et al. (1953) and Käfer (1958). 


\section{RESULTS}

\section{Carbohydrate utilization by the roild-type}

A brief preliminary survey of the growth of Aspergillus nidulans on a variety of organic compounds as sole carbon source was made by use of an auxanographic technique (Pontecorvo, 1949). A thick suspension of wild-type conidia in basal medium was poured in a series of Petri dishes, and these incubated for $18 \mathrm{hr}$. at $37^{\circ}$. Crystals of the various compounds were then placed at points on the surface of the agar and the resulting growth compared after a further $24 \mathrm{hr}$. of incubation. Good growth was obtained with D-glucose, D-fructose, sucrose, maltose, cellobiose, acetate, glycerol and D-sorbitol; moderate growth with mannose, D-galactose, rhamnose, melibiose, lactose, trehalose, raffinose, soluble starch, tartrate, erythritol and mannitol; L-sorbose, citrate and adonitol were not utilized. The carbohydrates selected for mutant screening experiments (Table 1) were chosen as effective but inexpensive carbon sources. Galactose, lactose and sorbitol are utilized after a period of adaptation.

\section{Mutants isolated}

The results of a number of mutant screening experiments are summarized in Table 1. The initial plates bore between 110 and 170 colonies each, and auxotrophic mutants (unable to grow on minimal medium but growing on complete medium) were isolated from them at a rate of $5 \cdot 35$ auxotrophs/1000 colonies. Mutants which did not grow on galactose occurred most frequently, but a few mutants were

Table 1. Mutants in Aspergillus nidulans failing to utilize specific carbohydrates for growth

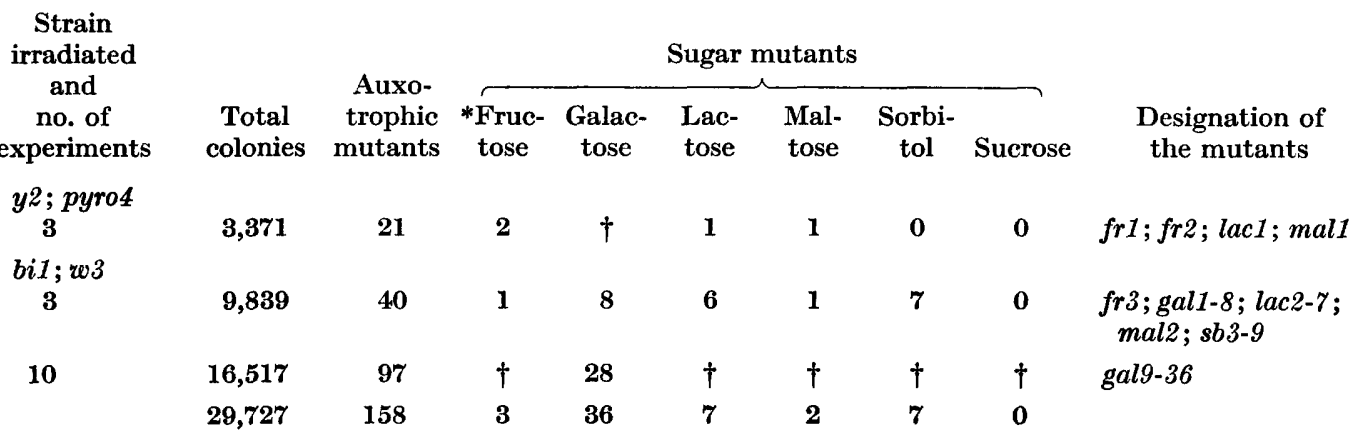

The mutants were identified by replica plating followng u.v.-irradiation (survival rates $4 \cdot 4-10 \cdot 7 \%$ ) by their failure to grow on a given sugar as sole carbon source.

* The mutants were isolated by their failure to grow on either sorbitol or sucrose.

$\dagger$ Not tested.

obtained for each of the other sugars tested except sucrose. The mutants were tested for their ability to grow on a variety of sugars as sole carbon source; it was generally found that they were unable to utilize one particular sugar. Three exceptions, involving fructose, maltose and some of the galactose mutants, are described below. Mutants of the type described in Escherichia coli which fail to grow on glucose but grow on a number of other sugars, including maltose (Doudoroff et al. 1949), would have been detectable in these experiments, but none was found. 


\section{Location and characteristics of the mutants}

The results of the analyses of the mutants are summarized in Table 2 which describes the loci identified, and Fig. 1 in which the position of loci in the genetic maps of the organism are shown. The mutants showed one of three characteristic phenotypes when tested for growth on the relevant sugars. In one the conidia germinated but did not grow beyond this stage; these are described as 'total mutants'. In a second phenotype the conidia germinated and formed very sparse colonies which produced a few conidial heads, the colonies increased in diameter at about the same rate as the wild type, but the density of the mycelium was greatly decreased. This 'starvation' growth is characteristic of Aspergillus nidulans when growth is limited by certain nutrients (Professor G. Pontecorvo, personal communication). Mutants of this second sort are described as 'partial mutants'.

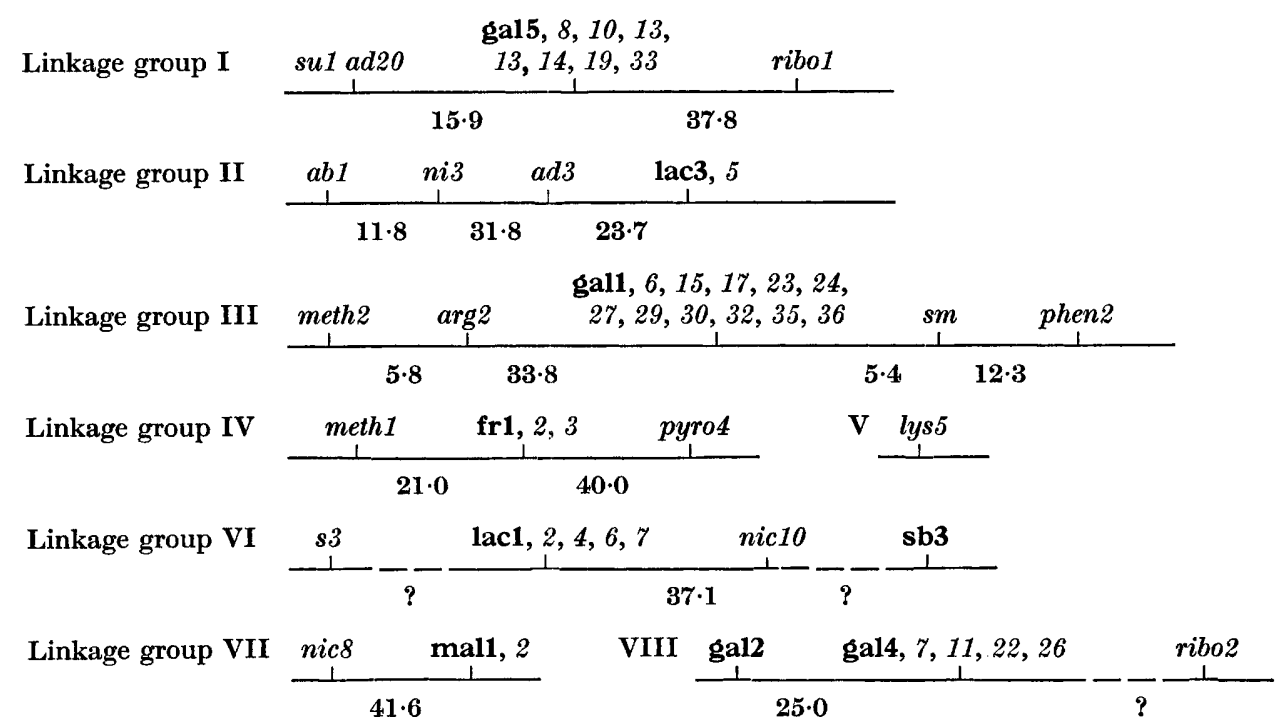

Fig. 1. Location of new markers (heavy type) in Aspergillus nidulans. Distances in meiotic recombination percentage. Symbols of mutant alleles: requirements, ribo1, ribo $2,=$ riboflavin $; a b 1=\gamma$-aminobutyric acid; $n i 3=$ nitrite; $a d 3=$ adenine; meth 1 , meth $2=$ methionine $; \arg 2=$ arginine $;$ phen $2=$ phenylalanine $;$ pyro $4=$ pyridoxin; lys $5=$ lysine; $s 3=$ sulphite; nic8, nic10 = nicotinic acid. su1 ad $20=$ suppressor of ad20; sm = small colony. (See Pontecorvo et al. 1953; Käfer, 1958, for further details.)

In mutants of a third phenotype the conidia germinated and produced morphologically abnormal colonies which grew slowly as compared to the wild type and formed few or no conidial heads. Only a few mutants of this type, called 'slow growing mutants', were isolated and these were all galactose mutants. Among the mutants isolated, those at a particular locus all tended to have the same phenotype (Table 2). The mutant characters usually segregated 1:1 from the wild type both at meiosis and in mitotic haploidization. They are therefore determined by differences at single loci. The mutants are all recessive to wild type both in diploids and in heterokaryons. 
Table 2. Loci controlling carbohydrate utilization identified in Aspergillus nidulans

\begin{tabular}{|c|c|c|c|c|}
\hline Locus* & Phenotype† & $\begin{array}{l}\text { Linkage } \\
\text { group }\end{array}$ & $\begin{array}{l}\text { No. of } \\
\text { mutants }\end{array}$ & Allelic mutants \\
\hline gal1 & $\begin{array}{l}\text { Utilization of: } \\
\text { Galactose }\left({ }^{\prime} \mathrm{T}\right)\end{array}$ & III & 12 & $\begin{array}{l}\text { gal } 6,15,17,23,24,27,29, \\
30,32,35,36\end{array}$ \\
\hline gal2 & Galactose (S) & VIII & 1 & - \\
\hline gal3 & Galactose (S) & II & $\mathbf{1}$ & - \\
\hline gal4 & Galactose (S) & VIII & $\mathbf{5}$ & gal7, $11,22,26$ \\
\hline gals & Galactose (T) & $\mathbf{I}$ & 7 & gals, 10, 13, 14, 19, 33 \\
\hline lac1 & Lactose (P) & VI & 5 & $\operatorname{lac} 2,4,6,7$ \\
\hline $\operatorname{lac} 3$ & Lactose (P) & II & 2 & lac5 \\
\hline mal1 & Maltose (P) & VII & 2 & mal2 \\
\hline$s b 3$ & Sorbitol (P, T) & VI & $\overline{7}$ & See text \\
\hline fr1 & Fructose (T) & IV & 3 & fr 2,3 \\
\hline
\end{tabular}

* The symbols of the loci represent the sugars which the mutants fail to utilize.

$\dagger \mathbf{T}=$ total mutant, $\mathbf{P}=$ partial mutant, $\mathbf{S}=$ slow-growing mutant (see text).

Ten galactose mutants (gal9, 12, 16, 18, 20, 21, 25, 28, 31, 34) recombine freely with gal1, gal4 and gal5; they have not been tested in crosses with gal2 or gal3.

Table 3. Growth of galactose mutants in Aspergillus nidulans on galactose and lactose

\begin{tabular}{lll} 
Mutant & \multicolumn{2}{c}{ Carbon source } \\
\cline { 2 - 3 } $\begin{array}{l}\text { Galactose } \\
\text { gal2; gal6 } \\
\text { gal4; gal } 3\end{array}$ & No growth & Lactose \\
gal5; gal8 & Slow growing & Wild type \\
& No growth & Partial growth
\end{tabular}

Table 4. Complementarity tests of eight galactose mutants in diploids heterozygous for pairs of gal mutants in trans arrangement

\begin{tabular}{|c|c|c|c|c|c|c|c|}
\hline & gal2 & gal3 & gal4 & gal5 & gal6 & gal7 & gals \\
\hline gal1 & + & + & + & + & - & + & + \\
\hline gal2 & . & + & + & + & $(+)$ & + & + \\
\hline gal3 & . & . & + & + & $(+)$ & + & + \\
\hline gal4 & . & . & . & + & $(+)$ & $(+)$ & + \\
\hline gal5 & . & . & . & . & $(+)$ & + & \\
\hline gal6 & . & . & . & . & $\cdot$ & $(+)$ & $(t$ \\
\hline gal7 & . & & . & . & . & $\cdot$ & + \\
\hline
\end{tabular}

$+=$ Wild-type growth: complementary mutants.

$(+)=$ Partial growth: partially complementing mutants.

$-=$ No growth: non-complementary mutants.

Groups of mutants between which there is complementation

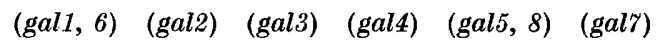

\section{Galactose mutants}

Eight galactose mutants isolated in a first series of u.v.-irradiation experiments may be separated into three phenotypic groups according to their growth on galactose or lactose as shown in Table 3. One possible explanation of the poor growth of 
gal 5 and gals on lactose, though by no means the only one, is an inhibition of growth by galactose similar to that described in strains of Escherichia coli lacking phosphogalactose transuridylase and accumulating Gal-1- $\mathrm{PO}_{4}$ (Yarmolinsky, Weismeyer, Kalckar \& Jordan, 1959).

Complementarity tests were done by synthesizing heterozygous diploids between the mutants taken in all possible pairwise combinations, using strains of the type paba1 $y a d 20 ; g a l_{x}$ and $b i 1 ; w 3 ; g a l_{y}$. The results of the tests are shown in Table 4.

Only two pairs of mutants (gal1 and gal6; gal5 and gal8) are completely noncomplementary, one pair (gal4 and gal7) is partially complementary, and gal6 is partially complementary in all combinations. The significance of the last result is not clear but the first two permit the mutants to be arranged in six complementing groups.

In addition, certain pairs of mutants were crossed using strains of the type paba1 y ad20; $g_{a l}$ and bi1; w3; gal $l_{y}$. From each cross a suspension of ascospores from a single hybrid perithecium was plated on complete medium in dilutions yielding about 25 colonies per dish. Approximately 100 colonies from each cross were tested individually for their growth on galactose.

No recombinants were detected among progeny derived from the cross between the two partially complementing mutants (gal4 and galy) which are assumed to represent a single locus. The remaining groups of complementing mutants recombine at readily detectable frequencies and therefore the eight galactose mutants define five loci controlling galactose utilization. In one case $($ gall $\times$ gal2) the segregation ratio (91 mutant: 13 wild) was significantly greater than 3 mutant: 1 wild, indicating linkage between gal2 and gal\%.

In later u.v.-irradiation experiments (see Table 1) a further 28 galactose mutants were isolated and these have been tested semi-quantitatively for linkage to gal1, gal4 or gal5 by means of a standard technique used in this laboratory. Crosses were set up between the new mutants and the three located mutants, hybrid perithecia selected and samples of ascospores streaked on galactose medium. The resulting growth was clearly either mutant or wild type and as each sample contained of the order of 200 ascospores the test could detect recombination down to about $1 \%$ between the known galactose mutants and the new mutants. At this level of analysis 10 of the 28 new mutants were found not to recombine with gall, 3 with gal4 and 5 with gal5 (Table 2). The remaining 10 new mutants which recombined freely with gal1, gal4 and gal5 have not been tested in crosses with gal2 or gal3.

The gall locus. The segregation of markers in haploid strains derived by mitotic haploidization from the diploid between a tester strain (Forbes, 1959) and bi1; w3; gal1 showed that gal1 is located in linkage group III. This result was confirmed by meiotic analysis (Table 5): gal1 is between arg2 and $s m$, and shows about $34 \%$ recombination with the former and about $5 \%$ with the latter.

The gal2 and gal4 loci. Mitotic haploidization, using a tester strain, suggests that gal4 is located in group VIII. However, the number of haploids tested is small and the result requires confirmation. Haploidization shows that gal2, which is linked to gal4, is not located in groups I, II, III, IV, V or VII, but no tests have been made with a tester strain marked in groups VI or VIII. Neither gal2 nor gal4 has been tested for meiotic recombination with group VIII markers, and location of these markers in group VIII is provisional. 
The gal3 locus. Mitotic haploidization located gal3 in linkage group II. Crosses involving gal3 and $w 3$, also in linkage group II, showed no linkage between these loci.

The gal5 locus. Mitotic haploidization located gal5 in linkage group I. This is confirmed by meiotic analysis (Table 6), and the locus is between ribo1 and su1 ad20, showing about $38 \%$ recombination with the former and about $16 \%$ with the latter.

Table 5. Location of gall in linkage group III by meiotic analysis

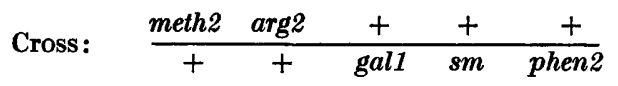

Interval: (1) (2) (3) (4)

Genotype of progeny

\begin{tabular}{|c|c|c|c|c|}
\hline meth & arg & + & + & + \\
\hline+ & + & gal & $s m$ & phen \\
\hline$\stackrel{+}{+}$ & $\begin{array}{c}\arg \\
+\end{array}$ & $\stackrel{+}{\text { gal }}$ & $\begin{array}{c}+ \\
s m\end{array}$ & $\stackrel{+}{+}$ \\
\hline$\stackrel{+}{+}$ & $\begin{array}{c}+ \\
\text { arg }\end{array}$ & $\stackrel{+}{g a l}$ & $\begin{array}{c}+ \\
s m\end{array}$ & $\stackrel{+}{\text { phen }}$ \\
\hline$\underset{\text { meth }}{+}$ & $\stackrel{+}{\arg }$ & $\begin{array}{c}\text { gal } \\
+\end{array}$ & $\begin{array}{c}+ \\
s m\end{array}$ & $\stackrel{+}{+}$ \\
\hline$\stackrel{+}{\text { meth }}$ & $\underset{\arg }{+}$ & $\begin{array}{c}\text { gal } \\
+\end{array}$ & $\begin{array}{c}s m \\
+\end{array}$ & $\begin{array}{c}+ \\
\text { phen }\end{array}$ \\
\hline $\begin{array}{c}\text { meth } \\
+\end{array}$ & $\stackrel{+}{\arg }$ & $\stackrel{+}{\text { gal }}$ & $\stackrel{+}{s m}$ & $\stackrel{+}{\text { phen }}$ \\
\hline $\begin{array}{c}\text { meth } \\
+\end{array}$ & $\stackrel{+}{\arg }$ & $\begin{array}{c}\text { gal } \\
+\end{array}$ & $\underset{s m}{+}$ & $\begin{array}{c}+ \\
\text { phen }\end{array}$ \\
\hline $\begin{array}{c}\text { meth } \\
+\end{array}$ & $\stackrel{+}{\arg }$ & $\begin{array}{c}\text { gal } \\
+\end{array}$ & $\begin{array}{c}s m \\
+\end{array}$ & $\stackrel{+}{+}$ \\
\hline $\begin{array}{c}\text { meth } \\
+\end{array}$ & $\begin{array}{c}\arg \\
+\end{array}$ & $\begin{array}{c}\text { gal } \\
+\end{array}$ & $\begin{array}{c}+ \\
s m\end{array}$ & $\stackrel{+}{+}$ \\
\hline $\begin{array}{c}\text { meth } \\
+\end{array}$ & $\begin{array}{c}\arg \\
+\end{array}$ & $\begin{array}{c}\text { gal } \\
+\end{array}$ & $\begin{array}{c}s m \\
+\end{array}$ & $\stackrel{+}{+}$ \\
\hline $\begin{array}{c}\text { meth } \\
+\end{array}$ & $\begin{array}{c}\arg \\
+\end{array}$ & $\stackrel{+}{\text { gal }}$ & $\begin{array}{c}s m \\
+\end{array}$ & $\begin{array}{c}+ \\
\text { phen }\end{array}$ \\
\hline$\stackrel{+}{+}$ & $\begin{array}{l}\text { arg } \\
+\end{array}$ & $\begin{array}{c}\text { gal } \\
+\end{array}$ & $\begin{array}{c}+ \\
s m\end{array}$ & $\stackrel{+}{\text { phen }}$ \\
\hline$\stackrel{+}{\text { meth }}$ & $\begin{array}{c}\text { arg } \\
+\end{array}$ & $\begin{array}{c}\text { gal } \\
+\end{array}$ & $\begin{array}{c}s m \\
+\end{array}$ & $\begin{array}{c}+ \\
\text { phen }\end{array}$ \\
\hline$\stackrel{+}{\text { meth }}$ & $\begin{array}{l}\text { arg } \\
+\end{array}$ & $\underset{\text { gal }}{+}$ & $\begin{array}{c}s m \\
+\end{array}$ & $\begin{array}{c}+ \\
\text { phen }\end{array}$ \\
\hline$\stackrel{+}{+}$ & $\stackrel{+}{\arg }$ & $\stackrel{+}{g a l}$ & $\begin{array}{c}s m \\
+\end{array}$ & $\stackrel{+}{+}$ \\
\hline $\begin{array}{c}\text { meth } \\
+\end{array}$ & $\stackrel{+}{\arg }$ & $\stackrel{+}{g a l}$ & $\begin{array}{c}s m \\
+\end{array}$ & $\begin{array}{c}+ \\
\text { phen }\end{array}$ \\
\hline
\end{tabular}

Class

\section{Parental}

Single cross-overs in (1)

Single cross-overs in (2)

Single cross-overs in (3)

Single cross-overs in (4)

Double cross-overs in (1) and (2)

Double cross-overs in (1) and (3)

Double cross-overs in (1) and (4)

Double cross-overs in (2) and (3)

Double cross-overs in (2) and (4)

Double cross-overs in (3) and (4)

Triple cross-overs in (1), (2) and (3)

Triple cross-overs in (1), (2) and (4)

Triple cross-overs in (1), (3) and (4)

Triple cross-overs in (2), (3) and (4)

Multiple cross-overs in (1), (2), (3) and (4)

Recombination fractions (\%):

$\begin{array}{lr}\text { gal1-meth2 } & 39 \cdot 5 \pm \mathbf{3} \cdot 0 \\ \text { gal1-arg2 } & 33 \cdot 8 \pm 2 \cdot 9 \\ \text { gal1-sm } & 5 \cdot 4 \pm 1 \cdot 6 \\ \text { gal1-phen2 } & 15 \cdot 8 \pm \mathbf{2} \cdot 3\end{array}$

$\begin{array}{lr}\text { meth2-arg2 } & 5 \cdot 8 \pm 1 \cdot 7 \\ \text { arg2-sm } & 35 \cdot 4 \pm 2 \cdot 9 \\ \text { sm-phen2 } & 12 \cdot 3 \pm 2 \cdot 0\end{array}$


Table 6. Location of gal5 in linkage group 1 by meiotic analysis

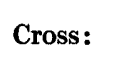

Interval:

Genotype of progeny

\begin{tabular}{|c|c|c|}
\hline su ad & gal & ribo \\
\hline+ & + & + \\
\hline+ & gal & ribo \\
\hline su $a d$ & + & + \\
\hline+ & + & ribo \\
\hline su $a d$ & gal & + \\
\hline su ad & + & ribo \\
\hline+ & gal & + \\
\hline & & Total \\
\hline
\end{tabular}

Recombination fractions (\%)

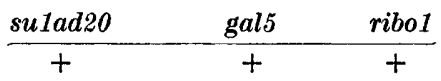

(1)

(2)

Class

Parental

Single cross-overs in (1)

Single cross-overs in (2)

Double cross-overs in (1) and (2)

Table 7. Location of lac1 in linkage group VI by mitotic haploidization

Classification of haploid strains isolated from the diploid

Tester $A / b i 1$; zo3; lac1

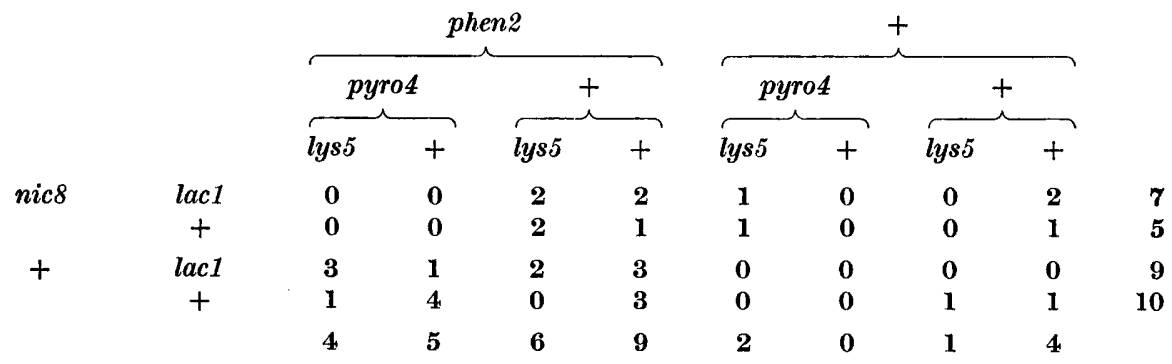

\begin{tabular}{rccrr}
\multicolumn{5}{c}{ Tester $C / b i 1 ;$; w $;$ lac1 } \\
s3 & \multicolumn{5}{c}{ ribo2 } & + & \\
& lac1 & 0 & 0 & 0 \\
+ & + & 3 & 6 & 9 \\
& lac1 & 8 & 11 & 19 \\
+ & 0 & 0 & 0
\end{tabular}

Tester strains contain markers identifying linkage groups. Tester A has markers in groups III (phen2), IV (pyro4), V (lys5) and VII (nic8); Tester C in groups VI (s3) and VIII (ribo2). A new marker is allocated to a linkage group by its failure to recombine with the marker defining the linkage group in haploid strains arising by mitotic haploidization from the diploid synthesized between a tester strain and a strain carrying the new marker. In this case lac1 recombines freely with all the markers tested except s3. Absence of recombinants of the type $s 3$ lac1 or $s 3^{+} l a c 1^{+}$ is expected if $s 3$ and $l a c 1$ are linked and in repulsion in the original diploid. This technique is fully described by Pontecorvo \& Käfer (1958) and by Forbes (1959). 


\section{Lactose mutants}

Seven phenotypically similar partial mutants were isolated and these classified into two complementing groups, which identify two loci, called lac1 and lac3, controlling the utilization of lactose (Table 2).

The lac1 locus. Mitotic haploidization located lac1 in linkage group VI which is defined by the marker $s 3$ (Table 7). In a cross including four group VI markers weak linkage was detected ( $37 \%$ recombination) between lac1 and nic10, but not between either of these markers and $s 3$ or $s b 3$ (Table 8 ).

Table 8. Location of lac1 in linkage group VI by meiotic analysis

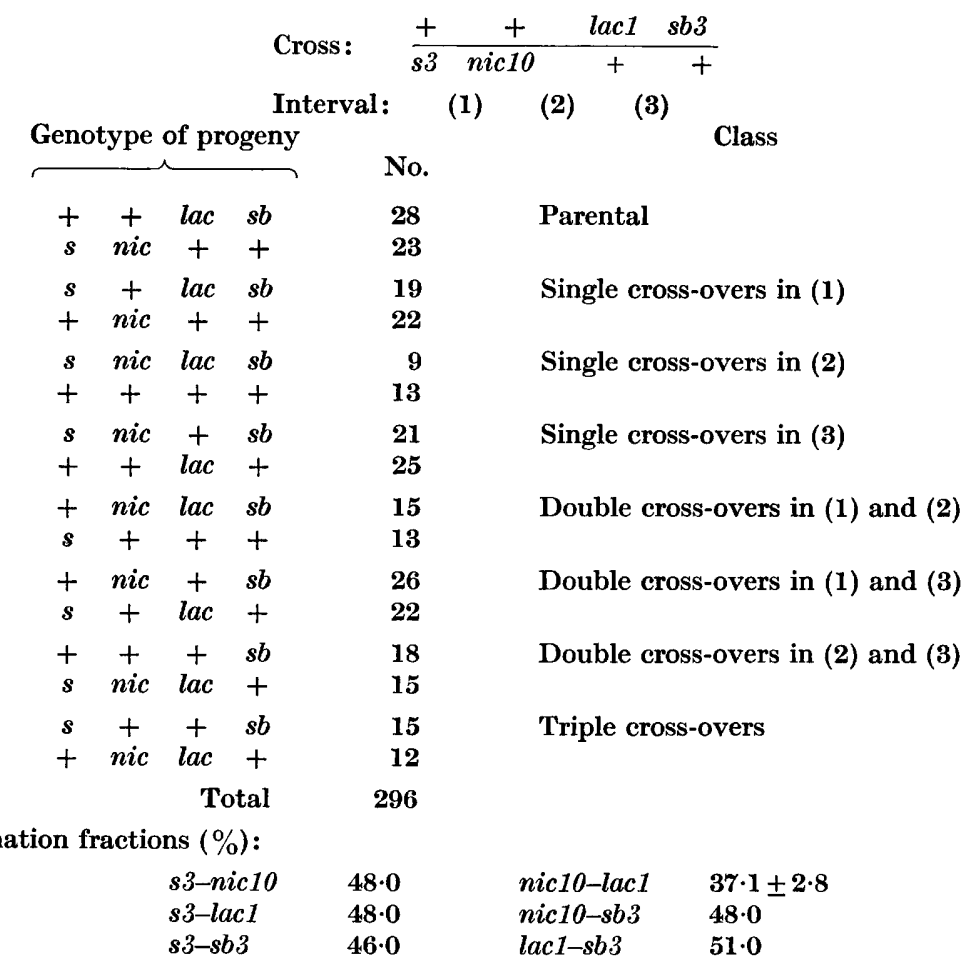

The lac3 locus. The mutant, lac5, which is non-complementary to lac3, was used in mapping this locus. Mitotic haploidization located lac5 in linkage group II. Three crosses involving altogether 8 group II markers, including the nutritional markers ribo6 and $a b 1$, located by other workers in group II but not mapped, were analysed in attempting to locate lac5. The two nutritional markers were located successfully and no linkage was detected between lac 5 and the following markers: ad23, Acr1, w3, ribo6, pu1, ab1 or ni3 (Fig. 2). In one cross weak linkage was detected between $a d 3$ and lac 5 but both these markers showed very poor viability. If it be assumed that the viability factors operate independently in the double mutant ad3 lac5, the recombination fraction can be estimated after calculating the values of the viability factors. (I am indebted to Dr J. S. Gale for suggestions about 
these calculations.) In this way a value of $23.7 \%$ was obtained for the recombination fraction between these two loci. It is desirable that location of lac 5 should be confirmed by analysis of further crosses particularly including the marker acr2.

Recombination fractions (\%) (Käfer, 1958):

New data:

Cross 1

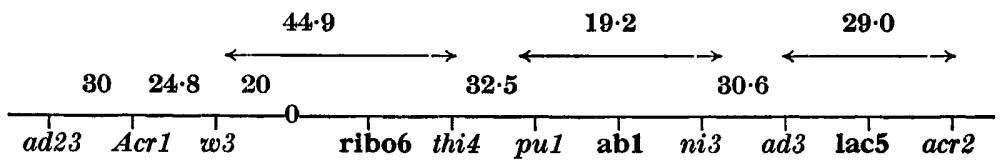

Cross 2
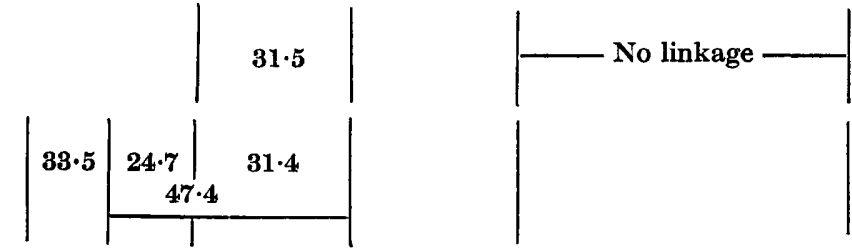

Cross 8
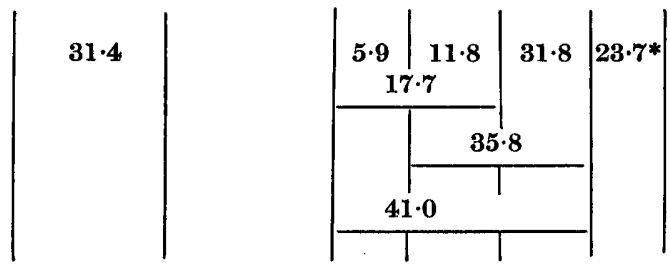

Fig. 2. Location of lac5 in linkage group II by meiotic analysis. The vertical bars indicate markers present in the crosses. The newly mapped markers are in heavy type.

* This recombination fraction is calculated on the assumption that there is no viability interaction between $a d 3$ and lac 5 (see text). Symbols of mutant alleles: requirements, ad23, $a d 3=$ adenine; ribo6 $=$ riboflavin; thi $4=$ thiamine; $p u 1=$ putrescine. Drug resistance, $\operatorname{Acr} 1$ (dominant), acr2 (recessive) = resistance to acriflavine; $w 3$ = white conidia. See also Fig. 1.

Table 9. Location of mall in linkage group VII by mitotic haploidization Classification of haploid strains isolated from the diploid

Tester $A / b i 1$; w3; mal1

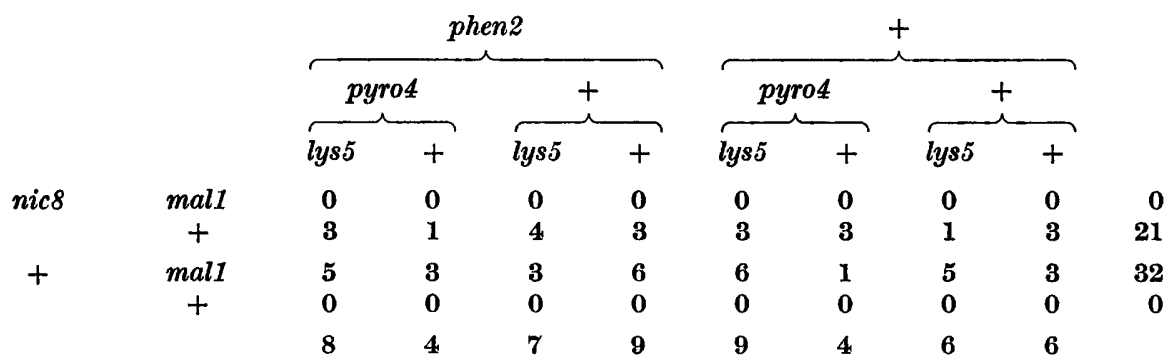

See Table 7 for explanation.

\section{Maltose mutants}

Both the maltose mutants isolated grow poorly on sucrose, which suggests that they are defective in $\alpha$-glucosidase. The mutants are non-complementary and identify a locus located in linkage group VII by mitotic haploidization (Table 9 ). 
Weak linkage $(4.1 \cdot 6 \%$ recombination) was detected between mal1 and nic 8 in a cross between these markers.

\section{Fructose mutants}

Three fructose mutants were isolated: one $(f r 1)$ was detected by failure to grow on sucrose and was originally given the symbol suc1 (this symbol was used by Forbes, 1959), and the other two ( $f r 2$ and $f r 3$ ) by failure to grow on sorbitol and originally called $s b 1$ and $s b 2$, respectively. All three mutants are identical phenotypically in that they fail to grow on either fructose, sorbitol or sucrose. The mutants are apparently inhibited by fructose or a metabolic product of fructose, for they fail to grow on glucose or other sugars to which small quantities of fructose have been added, and fail to utilize carbohydrates which either contain a fructose residue, such as sucrose, or are metabolized via fructose as in the case of sorbitol (Shockley \& Pride, 1959).

The three mutants are non-complementary and define a single locus concerned with fructose utilization which was located in linkage group IV by means of haploidization of diploids and mapped meiotically between meth1 and pyro4 (Table 10).

\section{Table 10. Location of fr1 in linkage group IV by meiotic analysis}

\section{Cross :}

Interval :

Genotype of progeny

$\begin{array}{ccccc}\text { Genotype of progeny } & \\ \text { meth } & + & \text { pyro } & \\ + & f r & + & \\ + & + & \text { pyro } & & \\ \text { meth } & f r & + & & \\ + & \text { fr } & \text { pyro } & & \\ \text { meth } & + & + & & \\ \text { meth } & f r & \text { pyro } & & \\ + & + & + & & 1 \\ & & \text { Total }\end{array}$

Recombination fractions (\%):

$\begin{array}{ll}\text { meth1-fr1 } & \mathbf{2 1} \cdot 0 \pm \mathbf{2 \cdot 3} \\ \text { fr1-pyro4 } & \mathbf{4 0 \cdot 0} \pm \mathbf{2 \cdot 8} \\ \text { meth1-pyro4 } & \mathbf{4 3 \cdot 5} \pm \mathbf{3 \cdot 1}\end{array}$

\section{Sorbitol mutants}

The sorbitol mutants are distinguishable from the fructose mutants by their ability to grow on sucrose and fructose. The seven mutants isolated are of two types; two are total mutants $(s b 3, s b 5)$ and the remaining five, partial mutants $(s b 4, s b 6$, $s b 7, s b 8, s b 9)$. In the present state of analysis a number of ambiguities have not been resolved and the relationship of the mutants has not been established.

The mutants were combined in all possible pairs in heterozygous diploids synthesized between strains of the type $b i 1 ; w 3 ; s b_{x}$ and $a d 14$ paba1 $y$; $s b_{y}$. When the diploids are tested for their growth on sorbitol three pairs of mutants are noncomplementary ( $s b 3$ and $s b 5 ; s b 4$ and $s b 7 ; s b 6$ and $s b 9)$ but complement in all other 
combinations, while $s b 8$ is non-complementary in all combinations. This result is described by the primitive complementation map shown in Fig. 3. Three of the mutants $(s b 3, s b 4, s b 9)$ were located in linkage group VI by mitotic haploidization (Table 11). Tests of $s b 3$ in crosses involving three other group VI markers (Table 8)$s 3$, nic10 and lac1-showed free recombination at meiosis between the $s b$ locus and all three others.

\begin{tabular}{cc}
$s b 8$ \\
\hline$s b 3, s b 5 \quad s b 4, s b 7 \quad s b 6, s b 9$
\end{tabular}

Fig. 3. Complementation of sorbitol mutants in heterozygous diploids of Aspergillus nidulans.

Table 11. Location of $\mathrm{sb3}, \mathrm{sb4}$ and $\mathrm{sb6}$ in linkage group VI by mitotic haploidization

\begin{tabular}{|c|c|c|c|c|c|c|c|c|c|c|}
\hline \multicolumn{11}{|c|}{$\begin{array}{l}\text { Classification of haploid strains isolated from the diploids } \\
\text { Tester } C / b i 1 ; w 3 ; s b 3, s b 4 \text { and } s b 9\end{array}$} \\
\hline & & ribo 2 & + & & & & & ribo2 & + & \\
\hline$s .3$ & $\begin{array}{l}s b 3 \\
+\end{array}$ & $\begin{array}{l}0 \\
4\end{array}$ & $\begin{array}{l}0 \\
1\end{array}$ & $\begin{array}{l}0 \\
5\end{array}$ & & $s 3$ & $\begin{array}{c}s b 4 \\
+\end{array}$ & $\begin{array}{r}0 \\
17\end{array}$ & $\begin{array}{l}0 \\
8\end{array}$ & $\begin{array}{r}0 \\
25\end{array}$ \\
\hline+ & $\begin{array}{l}s b 3 \\
+\end{array}$ & $\begin{array}{l}4 \\
0\end{array}$ & $\begin{array}{l}2 \\
0\end{array}$ & $\begin{array}{l}\mathbf{6} \\
\mathbf{0}\end{array}$ & & + & $\begin{array}{l}s b 4 \\
+\end{array}$ & $\begin{array}{r}12 \\
0\end{array}$ & $\begin{array}{l}\mathbf{9} \\
\mathbf{0}\end{array}$ & $\begin{array}{r}21 \\
0\end{array}$ \\
\hline & & 8 & 3 & & & & & 29 & 17 & \\
\hline & & & & & ribo2 & + & & & & \\
\hline & & & 83 & $\begin{array}{c}s b 9 \\
+\end{array}$ & $\begin{array}{l}0 \\
1\end{array}$ & $\begin{array}{l}\mathbf{0} \\
\mathbf{3}\end{array}$ & $\begin{array}{l}0 \\
4\end{array}$ & & & \\
\hline & & & + & $\begin{array}{l}s b 9 \\
+\end{array}$ & $\begin{array}{l}7 \\
0\end{array}$ & $\begin{array}{l}\mathbf{5} \\
\mathbf{0}\end{array}$ & $\begin{array}{r}12 \\
0\end{array}$ & & & \\
\hline & & & & & 8 & 8 & & & & \\
\hline
\end{tabular}

See Table 7 for explanation.

In testing the sorbital mutants for functional relationships the unusual result was observed that although certain pairs of mutants complement in heterozygous diploids, none of the pairs of mutants complements in heterokaryons. Balanced heterokaryons were synthesized between pairs of strains of the type $b i 1 ; w 3 ; s b_{x}$ and $a d 14$ paba1 $y ; s b_{y}$. The heterokaryons were tested by transferring actively growing mycelium from minimal medium to a test medium of basal medium + sorbitol. They all showed partial growth, that is they were all mutant. Supplementing this test medium with nutrients required by the component strains, either altogether or in various combinations, did not appreciably improve the growth of the mycelium. Variation of the incubation temperature was also without effect. The heterokaryotic mycelia continued to show partial (mutant) growth for at least three serial subcultures on unsupplemented basal medium + sorbitol but always immediately yielded vigorously growing heterokaryons on return to minimal medium. That the difference between complementarity of pairs of sorbitol mutants in heterokaryons and in heterozygous diploids is a function of the $s b$ mutants themselves, is shown by the fact that it appears also between other heterokaryons and diploids with the same combinations of $s b$ mutants but with different combinations of nutritional markers, namely ad14 paba1 $y$ and ribo1 pro1 bi1; w3. 


\section{DISCUSSION}

This paper emphasizes the usefulness of the new type of mutants in mould genetics. In Aspergillus nidulans the mutants segregate clearly in both meiotic and mitotic recombination, they usually have good viability, and they can be used in selective plating techniques.

The possibility that differences in phenotype may occur between combinations of the same genes in heterokaryons and in heterozygotes was predicted by Pontecorvo (1952 $b$ ) and has been discussed more recently by other authors (Roper, 1958; Lewis, 1961). Differences have been described in Ustilago in the pathogenicity and saprophytic growth of dikaryons as compared with diploids (Holliday, 1961) and also in the case of pan2 mutants of Neurospora combined in heterokaryons or in disomic 'pseudowild' strains (Case \& Giles, 1960). The series of sorbitol mutants described here, which are non-complementary in heterokaryons, although certain pairs complement in heterozygous diploids, constitutes a further example in $A$. nidulans. Two facts suggest that the difference is a property of the mutants rather than a nutritional interaction or the result of an unfavourable nuclear balance in the heterokaryons. First, the mutants do not complement in heterokaryons when the test medium is supplemented with mixtures of the nutrients required by the component strains, that is when provided with the opportunity for a shift in the nuclear ratio. Secondly, they also fail to complement when tested in combinations with different nutritional markers.

Recent work by the author has shown that the oxidation of sorbitol by intact organisms involves an inducible enzyme system; experiments to test for differences in enzyme formation between the heterokaryons and heterozygotes will be described in a later publication. It is clearly necessary to distinguish genetically between the possibilities that the sorbitol mutants represent three cistrons spanned by a deletion $(s b 8)$ or a single cistron within which complementation occurs between some alleles (Catcheside \& Overton, 1958; Catcheside, 1960). In the first case models could be built (Professor G. Pontecorvo, personal communication) in which the mutants involve regulating and structural cistrons (Jacob \& Monod, 1961) and failure of the mutants to complement across the cytoplasm results from differences in the local concentrations of the apo-repressor. If the mutants involve only structural cistrons, analogous to those which determine the structure of the $\mathbf{A}$ and $\mathbf{B}$ components of Escherichia coli tryptophan synthetase (Yanofsky, 1960), complementation may only occur in the heterozygotes as the result of interaction of gene products at a state intermediate between DNA and polypeptide-as suggested by Dorn \& Burdick (1962) for an example in Drosophila-rather than an interaction of protein subunits in the cytoplasm as is commonly held (see Catcheside, 1960). Phenomena of the sort described here may well prove more common as studies of the genetics of moulds are extended, and may be of interest in the study of protein synthesis and the mechanisms of intra-cistron complementation.

The author wishes to thank Professor G. Pontecorvo, F.R.S. for his advice and stimulating criticism. This work has been supported by the Nuffield Foundation and the Agricultural Research Council. 


\section{REFERENCES}

CASE, M. E. \& Giles, N. H. (1960). Comparative complementation and genetic maps of the pan2 locus in Neurospora crassa. Proc. nat. Acad. Sci., Wash. 46, 659.

Catcheside, D. G. (1960). Complementation among the histidine mutants of Neurospora crassa. Proc. Roy. Soc. B, 153, 179.

Catcheside, D. G. \& Overton, A. (1958). Complementation between alleles in heterokaryons. Cold Spr. Harb. Symp. quant. Biol. 23, 137.

Cohen, G. N. \& Monod, J. (1957). Bacterial permeases. Bact. Rev. 21, 169.

DorN, G. L. \& BURDick, A. B. (1962). On the recombinational structure and complementation relationships in the $m$-dy complex of Drosophila melanogaster. Genetics, 47, 503.

Doudoroff, M., Hassid, W. Z., Putman, E. W., Potter, A. L. \& Lederberg, J. (1949). Direct utilisation of maltose by Escherichia coli. J. biol. Chem. 179, 921.

Elurotr, C. G. (1960). The cytology of Aspergillus nidulans. Genet. Res., Camb. 1, 462.

Emerson, S. (1944). The induction of mutations by antibodies. Proc. nat. Acad. Sci., Wash. 30, 179.

FonBes, E. (1959). Use of mitotic segregation for assigning genes to linkage groups in Aspergillus nidulans. Heredity, 13, 67.

Hemmons, L. M., Pontecorvo, G. \& Bufton, A. W. J. (1953). Perithecium analysis and relative heterothallism. Advanc. Genet. 5, 194.

Holliday, R. (1961). Induced mitotic crossing over in Ustilago maydis. Genet. Res., Camb. 2, 231.

JACOB, F. \& MONOD, J. (1961). Genetic regulatory mechanisms in the synthesis of proteins. J. mol. Biol. 3, 318.

KäFER, E. (1958). An 8-chromosome map of Aspergillus nidulans. Advanc. Genet. 9, 105.

LANDMAN, O. E. (1950). Formation of lactase in mutants and parental strains of Neurospora. Genetics, 35, 673.

LEWIS, D. (1961). Genetical analysis of methionine suppressors in Coprinus. Genet. Res., Camb. 2, 141.

MATHER, K. (1951). The Measurement of Linkage in Heredity, 2nd ed. London: Methuen.

Ponteconvo, G. (1949). Auxanographic techniques in biochemical genetics. J. gen. Microbiol. 3, 122.

Pontecorvo, G. (1952a). Genetic formulation of gene structure and function. Advanc. Enzymol. 13, 121.

Ponteconvo, G. (1952b). Genetical analysis of cell organisation. Symp. Soc. exp. Biol. 6, 218.

Pontecorvo, G. (1954). Mitotic recombination in the genetic systems of filamentous fungi. Caryologia, 6 (suppl.), 192.

Ponteconvo, G. (1958). Trends in Genetic Analysis. New York: Columbia University Press.

Pontecorvo, G. \& Käfer, E. (1958). Genetic analysis based on mitotic recombination. Advanc. Genet. 9, 71.

Pontecorvo, G., Roper, J. A., Hemmons, L. M., Macdonald, K. D. \& Bufton, A. W. J. (1953). The genetics of Aspergillus nidulans. Advanc Genet. 5, 141.

Pontecorvo, G., TARr Gloor, E. \& Forbes, E. (1954). Analysis of mitotic recombination in Aspergillus nidulans. J. Genet. 52, 226.

Roberts, C. F. (1959). A replica plating technique for the isolation of nutritionallyexacting mutants of a filamentous fungus (Aspergillus nidulans). J.gen. Microbiol. 20,540.

Roper, J. A. (1952). Production of heterozygous diploids in filamentous fungi. Experientia, 8, 14.

Roper, J. A. (1958). Nucleo-cytoplasmic interactions in Aspergillus nidulans. Cold Spr. Harb. Sym. quant. Biol. 23, 141.

Shockley, T. E. \& Pride, M. S. (1959). D-Sorbitol-6-phosphate dehydrogenase from Lactobacillus casei. J. Bact. 77, 695.

YANOFSKy, C. (1960). The tryptophan synthetase system. Bact. Rev. 24, 221.

Yarmolinsky, M. B., WeismeYer, H., Kalckar, H. M. \& Jordan, E. (1959). Hereditary defects in galactose metabolism of Escherichia coli. II. Galactose induced sensitivity Proc. nat. Acad. Sci., Wash. 45, 1786. 